\title{
Le Centre de Ressources en Langues et en Communications de Trinity College Dublin (République d'Irlande)
}

Imelda Bonel-Elliot

\author{
(2) OpenEdition \\ Journals \\ Édition électronique \\ URL : http://journals.openedition.org/edc/2410 \\ DOI : 10.4000/edc. 2410 \\ ISSN : 2101-0366 \\ Éditeur \\ Université de Lille \\ Édition imprimée \\ Date de publication : 1 décembre 1996 \\ Pagination : 41-46 \\ ISBN : 978-2-07-677030-8 \\ ISSN : $1270-6841$
}

Référence électronique

Imelda Bonel-Elliot, « Le Centre de Ressources en Langues et en Communications de Trinity College Dublin (République d'Irlande) », Études de communication [En ligne], 19| 1996, mis en ligne le 20 juin 2011, consulté le 19 mars 2021. URL : http://journals.openedition.org/edc/2410 ; DOI : https://doi.org/ $10.4000 /$ edc. 2410

Ce document a été généré automatiquement le 19 mars 2021.

(c) Tous droits réservés 


\title{
Le Centre de Ressources en Langues et en Communications de Trinity College Dublin ${ }^{1}$ (République d'Irlande)
}

\author{
Imelda Bonel-Elliot
}

1 En 1978, a été créé le Centre de Ressources en Langues et en Communications de Trinity College Dublin (CLCS), dans le cadre de la construction d'un nouveau bâtiment pour les Facultés de Lettres et de Sciences Humaines. Ce centre de ressources a trois objectifs principaux : accueillir les étudiants spécialistes en langues qui souhaitent utiliser les équipements en auto-apprentissage, assurer l'enseignement de linguistique (aux étudiants de premier et de deuxième cycles) et faire des recherches en linguistique théorique et appliquée, et enfin, dispenser un enseignement de langues personnalisé aux étudiants des autres disciplines.

\section{Le centre de ressources}

Laboratoires de langues : 33 cabines.

3 Salle de vidéo et de visionnement de programmes télévisés reliés par satellite : 15 magnétoscopes et postes de télévision. Espace multimédia : 14 ordinateurs (PC) dont 6 multimédia.

5 Salle de ressources: documents audio, vidéo et écrits. Des documents en version originale, des documents pédagogiques et des outils de formation.

Documents : nombreux documents vidéo en français, allemand, italien et espagnol ainsi que des documents audio et écrits en allemand, espagnol, français, gaélique, italien et russe. Un plus petit nombre de documents audio et écrits pour une vingtaine d'autres langues. 

Ressources a été mise en place par un des chercheurs du centre. Cette base de données permet aux étudiants de trouver des informations pédagogiques sur l'ensemble des produits disponibles.

\section{Le personnel :}

ix enseignants-chercheurs, neuf assistants/ingénieurs de recherche, six enseignants à mi-temps, une trentaine d'assistants à temps partiel, deux techniciens, deux responsables administratifs-archivistes.

\section{Les modules pour les étudiants d'autres disciplines}

11 En 1993, le Comité d'Enseignement Supérieur ${ }^{2}$ en République d'Irlande a annoncé la mise en place d'un projet pilote pour rénover certaines filières de premier et de deuxième cycles. L'un des objectifs était de mettre en place des modules de langues vivantes pour les étudiants en Lettres, en Commerce, en Sciences et dans les formations d'ingénieur. Le Centre de Ressources en Langues et en Communications de Trinity College Dublin a obtenu une somme de $£ 100000$ (environ 850000 francs) pour mettre en place des modules d'allemand pour les grands débutants, et des modules d'allemand et de français pour les étudiants ayant déjà étudié ces langues dans le secondaire (généralement pendant 5 ans). Le principe de base de ces modules était l'autoapprentissage guidé.

12 En 1993-1994, les étudiants avaient deux heures par semaine programmées pour leur module de langue. Pendant la première demi-heure, un professeur de la langue cible leur explique le travail à faire et les objectifs de ce travail. Ensuite les étudiants travaillent en groupe avec un assistant du pays de la langue cible pendant une demiheure, puis de manière autonome dans le Centre de Ressources en Langues et en Communications (à l'aide de la télévision par satellite, de la vidéo, de l'audio ou de l'informatique) pendant une autre demi-heure sous la surveillance d'un tuteur/ professeur disponible pour les aider sur leur demande. La dernière demi-heure est consacrée à la synthèse et la consolidation du travail effectué avec le professeur responsable.

13 Au bout d'un semestre, les modules ont été réorganisés pour tenir compte des critiques des étudiants et a été mis en place un cours d'une heure pour organiser le travail des étudiants. De plus, les assistants font des exposés courts sur des thèmes pointus en Sciences ou en Lettres, en fonction du cursus et de l'intérêt de l'étudiant. Ces exposés servent de base pour la compréhension orale, l'expression écrite et orale. Pendant la deuxième heure, les étudiants se divisent en petits groupes pour travailler avec les assistants ou dans le Centre de Ressources en autonomie guidée. Apparemment, les étudiants ressentent le besoin d'être bien encadrés pendant au moins une heure par semaine, le travail dans le Centre de Ressources étant utile dans le cadre d'un projet pédagogique bien conçu. 
14 Pour que le module de langue apporte des bénéfices durables aux jeunes, les responsables du Centre de Ressources se sont efforcés de rendre l'apprentissage le plus efficace possible. Dans ce but, ils ont essayé de rendre les apprenants autonomes en les encourageant à se sentir responsables de leur apprentissage et en les rendant très conscients du processus d'acquisition d'une langue vivante étrangère. En effet, sous la direction $d u$ directeur du centre, David Little, la coordinatrice du projet, en collaboration avec l'équipe pédagogique, a rédigé un document de six pages pour les étudiants qui explique dans le détail la manière dont ils doivent travailler et comment ils peuvent progresser en langue. Le carnet de l'apprenant fait partie intégrante de ce projet. Chaque semaine, l'apprenant remplit son carnet en explicitant son travail cible pour la semaine, le travail fourni chaque jour et les supports utilisés. Il fait une autoévaluation de son travail et de ses progrès. Ce carnet est utile pour les chercheurs du Centre, mais n'est pas utilisé pour l'évaluation sommative de l'apprenant.

Les responsables du Centre souhaitent encourager les étudiants à acquérir une capacité à apprendre une langue de manière indépendante pendant et après leurs études universitaires. L'apprentissage autonome semi-guidé leur semble utile à cette fin. L'avantage de l'auto-apprentissage est qu'il permet aux apprenants d'utiliser les équipements de manière individuelle et au moment où ils sont disponibles. La présence d'enseignants et d'autres personnes qualifiées paraît indispensable pour aider les apprenants à choisir une stratégie d'apprentissage, pour les aider à sélectionner les documents sonores, vidéo, iconographiques ou graphiques les plus appropriés à leurs besoins et à leurs centres d'intérêt.

Chaque année, le projet est évalué par une équipe d'experts externes. Leurs rapports sont globalement très positifs. Ces experts font remarquer que l'intérêt de cette démarche est qu'elle permet un apprentissage efficace des langues vivantes étrangères. L'accès à un matériel sophistiqué dans un centre de ressources en langues ne suffit pas pour apprendre une langue. Il faut un projet pédagogique bien conçu avec un personnel enseignant qualifié et expérimenté pour le mener à bien. Les évaluateurs externes attirent l'attention sur le fait que ces modules ne pourraient pas fonctionner de manière efficace avec des assistants sans l'encadrement des professeurs qualifiés et expérimentés, et que tout travail vraiment efficace a un coût que l'on ne peut pas réduire si l'on veut garder un travail de qualité.

\section{Evaluation}

17 L'évaluation des étudiants est fondée sur une épreuve écrite (celle-ci comprend une dictée et un exercice à trous) et une épreuve orale comprenant un exposé devant les examinateurs et devant les autres étudiants. En 1994, 98 \% des étudiants ont obtenu une note satisfaisante et $43 \%$ une mention. Il faut tout de même tenir compte du fait que ces modules sont des options facultatives, et que tous les étudiants ne les passent pas. Le taux est allé de 35,5\% - 1993-1994 - à $60 \%$ - 1994-1995. On peut, donc, constater que les étudiants doivent être très motivés pour obtenir une qualification en langue. Ces résultats figurent sur les diplômes, mais ne sont pas comptabilisés pour l'obtention de l'année d'études. Les étudiants peuvent également préparer des diplômes de langues reconnus sur le plan international s'ils le souhaitent. Dans les faits, une auto-sélection s'opère et seuls les plus motivés font ce travail supplémentaire. Bien que les compétences en langue étrangère puissent être un atout pour un jeune lors de la 
recherche d'un emploi, il faudra certainement longtemps avant que la majorité des étudiants maitrise au moins une langue de la communauté européenne. Cette expérience intéressante semble représenter un pas important dans ce sens.

\section{Recherches}

Le directeur du centre, David LITTLE, est un chercheur connu sur le plan international pour ses travaux sur l'apprentissage des langues vivantes, en particulier dans les domaines de l'autonomie de l'apprenant, de l'utilisation des documents authentiques et de l'emploi des technologies modernes. De 1981 à 1996, de nombreux articles sur la linguistique générale et appliquée et sur l'apprentissage des langues ont été publiés par le centre, dans la collection "Occasional Papers" dirigé par David LITTLE. Toute l'équipe de chercheurs est mobilisée, ainsi qu'en témoignent leurs publications et leur participation au colloque de CERCLES ${ }^{3}$ en septembre 1996. En outre, David LITTLE est directeur d'Authentik ${ }^{4}$, entreprise du campus ${ }^{5}$ qui se charge d'éditer et de publier des revues de qualité contenant des documents authentiques avec des exercices pédagogiques en allemand, en anglais, en espagnol et en français prévus pour les élèves du secondaire et pour les étudiants dans le supérieur.

Le Centre de Ressources en Langues et en Communications a également un contrat de recherche-développement d'une durée de trois ans pour développer les modules en langues étrangères. Les chercheurs vont faire des recherches en collaboration avec des experts de l'évaluation dans des universités allemandes pour définir et calibrer les niveaux en langues. Ils vont développer des modules dans des langues autres que le français et l'allemand (d'abord l'italien, puisqu'une étude détaillée des besoins en langues étrangères en 1994-1995 a montré une assez forte demande pour cette langue). Ils ont également l'intention de développer des produits pédagogiques informatisés, de la vidéo interactive et d'améliorer la base de données existante. De plus, ils vont continuer leurs recherches sur la motivation et l'aptitude des étudiants, et sur l'efficacité de l'informatique et de la vidéo interactive dans l'apprentissage des langues. Ils espèrent également éditer des produits pédagogiques afin que les établissements de l'enseignement supérieur qui souhaitent les utiliser puissent y avoir accès.

\section{Partenariat}

20 Le Centre de Ressources en Langues et en Communications participe à un projet européen coordonné par la Ruhr-Universität de Bochum pour explorer le rôle du courrier électronique dans l'apprentissage des langues étrangères. En effet, tous les étudiants de Trinity College Dublin ont leur code et leur accès personnel au courrier électronique dès leur inscription à l'Université.

\section{Conclusion}

Le Centre de Ressources en Langues et en Communications de Trinity College Dublin est un centre important de recherches et d'innovation dans le domaine de l'apprentissage des langues vivantes. Son grand intérêt est le fait que la recherche théorique et appliquée informe la pratique, et que la pratique sert de base également pour les 
recherches. Cette remise en cause continuelle permet l'innovation. Ce centre étant dans des bâtiments conçus il y a une vingtaine d'années, on ne peut pas créer un centre sur mesure comme les responsables le souhaiteraient, mais la force de l'équipe est peutêtre le fait qu'elle s'adapte et parvient à innover en dépit des contraintes d'un bâtiment assez ancien. Les modules de langues pour les étudiants d'autres disciplines ne représentent, bien sûr, qu'une partie du travail du Centre de Ressources en Langues et en Communications de Trinity College Dublin, mais leur caractère innovant est d'un grand intérêt.

\section{NOTES}

1. Centre for Language and Communication Studies (CLCS) Trinity College Dublin (TCD), Dublin 2, Irlande. Nous remercions le personnel du CLCS pour son concours lors de la préparation de cette présentation.

2. Le Comité d'Enseignement Supérieur ou « Higher Education Authority » (HEA) est chargé par l'Etat du financement de l'enseignement supérieur.

3. CERCLES = Confédération Européenne des Centres de Langues dans l'Enseignement Supérieur.

4. Authentik Language Learning Resources Ltd - 27 Westland Square - Dublin 2 - Irlande. Authentik fut fondée il y a une quinzaine d'années par Sean DEVITT du Département de Sciences de l'Education. Il s'agit d'une entreprise privée (comme n'importe quelle autre entreprise). Le fait que ce soit une entreprise du campus signifie que l'Université (Trinity College Dublin) est actionnaire et permet à deux enseignants, DavidLITTLE et Sean DEVITT, de faire une petite partie de leur service pour cette société. Authentik emploie actuellement six rédacteurs, quatre responsables de l'administration et du marketing et quelques personnes à temps partiel. Ses marchés se trouvent principalement en Irlande et en Grande Bretagne. La plupart des revues sont vendues directement aux établissements scolaires. Source : D.LITTLE et un conseiller fiscal du cabinet d'Ernst \& Young.

5. «Campus Company ».

\section{AUTEUR}

\section{IMELDA BONEL-ELLIOT}

Imelda Bonel-Elliott, Directrice du Département d'Anglais - Université du Littoral 